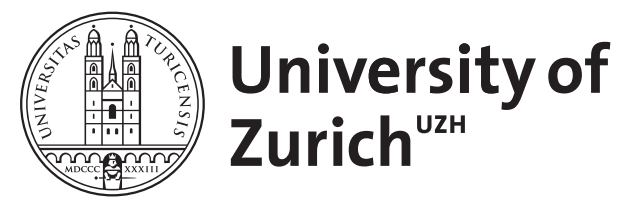

\title{
Immunotherapy for neurodegeneration?
}

\author{
Liu, Yingjun ; Aguzzi, Adriano
}

\begin{abstract}
Alzheimer's disease (AD), Parkinson's disease (PD), and prion diseases such as CreutzfeldtJakob disease attack different parts of the central nervous system (CNS) and elicit distinct symptoms, yet they share many biochemical and neuropathological features. These include the formation of protein aggregates in the affected brain regions and progressive activation of non-neuronal cells in the brain that play crucial roles in immune responses. The activation of immune cells in the CNS ("neuroinflammation") is prominent in these diseases. However, it remains unclear whether boosting or suppressing the immune system, in the brain or in the periphery, may attenuate neurodegeneration. In the case of extraneural prion infections, genetic or pharmacological ablation of components of the immune system, such as B cells and complement, can prevent disease (1). However, immunotherapies, which have been successful in treating certain types of cancer, have yet to reverse neurodegeneration in any patients. Therefore, the therapeutic promise of this approach remains debatable.
\end{abstract}

DOI: https://doi.org/10.1126/science.aaw0685

Posted at the Zurich Open Repository and Archive, University of Zurich

ZORA URL: https://doi.org/10.5167/uzh-171135

Journal Article

Accepted Version

Originally published at:

Liu, Yingjun; Aguzzi, Adriano (2019). Immunotherapy for neurodegeneration? Science, 364(6436):130131.

DOI: https://doi.org/10.1126/science.aaw0685 


\title{
Immunotherapy for neurodegeneration?
}

\author{
The role of innate and adaptive immunity in neurodegeneration remains controversial \\ By Yingjun Liu and Adriano Aguzzi
}

Alzheimer's disease (AD), Parkinson's disease (PD), and prion diseases such as Creutzfeldt-Jakob disease, attack different parts of the central nervous system (CNS) and elicit distinct symptoms - yet they share many biochemical and neuropathological features. These include formation of protein aggregates in the affected brain regions and progressive activation of non-neuronal cells in the brain that play crucial roles in immune responses. The activation of immune cells in the CNS ("neuroinflammation") is prominent in these diseases. However, it remains unclear whether boosting or suppressing the immune system, in the brain or in the periphery, may attenuate neurodegeneration. In the case of extraneural prion infections, genetic or pharmacological ablation of components of the immune system, such as B cells and complement, can prevent disease (1). However, immunotherapies, which have been successful in treating certain types of cancer, have yet to reverse neurodegeneration in any patients. Therefore, the therapeutic promise of this approach remains debatable.

Immunotherapy such as immune checkpoint blockade, which enhances the systemic adaptive immune response, may have potential in combating neurodegeneration, especially in $\mathrm{AD}(2,3)$. But recent studies have failed to observe any beneficial effects of immune checkpoint blockade in mouse models of $\mathrm{AD}(4)$ and of prion diseases (5, 6), raising questions about the effectiveness of this approach. Conversely, findings from studying human genetics and experimental models suggest that innate immunity in the brain has pivotal roles in the pathogenesis of neurodegenerative diseases. Therefore, targeting the immune reactions inside the brain may have enormous potential for treating these devastating disorders - yet a clear therapeutic path is amiss, and well-defined, validated targets are still in demand.

The inhibition of immune checkpoint receptors, an approach to fight cancers through boosting the adaptive immune system, has revolutionized cancer medicine (and was awarded a Nobel Prize in 2018). Immune

Institute of Neuropathology, University of Zurich, Schmelzbergstrasse 12, CH-8091 Zurich, Switzerland. Email:yingjun.liu@usz.ch; adriano.aguzzi@usz.ch checkpoint inhibition is mainly achieved by systemically blocking the activity of negative regulators of $\mathrm{T}$ cell activation, such as programmed cell death 1 (PD1), cytotoxic Tlymphocyte associated antigen 4 (CTLA4) and lymphocyte activation gene 3 (LAG3). The expression of these molecules, among others, establishes a state of immune suppression called tolerance towards cells of the body, which underlies the failure of the immune system to clear tumor cells (which are derived from cells of the body). Might systemic immune suppression contribute to the inefficient clearance of pathological protein aggregates in neurodegenerative diseases? Indeed, treatment with PD1-specific antibodies enhanced infiltration of peripheral myeloid cells into the CNS and reduced the amount of amyloid $\beta(A \beta)$ plaques in the hippocampus and cortex (two brain regions most affected in AD) in two transgenic mouse models of AD. Moreover, LAG3 was found to facilitate the cell-to-cell spread of $\alpha$ synuclein in a mouse model of PD (7).

Although PD1-specific antibodies are not harmless and can trigger autoimmunity, repurposing them for treating $\mathrm{AD}$ - an untreatable condition - could be justified. However, another study has found no effect of anti-PD1 antibody therapy on myeloid cell infiltration into the brain and $A \beta$ load in these CNS regions in three other $\mathrm{AD}$ mouse models (4).

The brain A $\beta$ load does not always correlate with cognitive performance, and the major determinant of cognitive decline is loss of synapses. Therefore, functional recovery tests deserve a high priority when evaluating therapeutic interventions for neurodegenerative diseases. Encouragingly, the clearance of $A \beta$ plaques was accompanied by recovery of cognitive performance in some of the antiPD1 antibody-treated mouse models of AD, although effects on synapse dynamics was not studied (2). In other AD mouse models however, the effects of anti-PD1 treatment on cognitive function was not investigated (4). Hence, the molecular underpinnings of the functional recovery in the successfully treated AD mouse models are still somewhat nebulous (see the figure).

Moreover, immune checkpoint blockade may affect cognition independently of plaque clearance. A plethora of immune modulators, including cytokines, complement components, and histocompatibility proteins play vital roles in synapse formation, refinement, and excitability, as well as in structural and functional synaptic plasticity during development and in the adult brain (8) Treatment with anti-PD1 antibodies may induce the expression of these immune modulators and their entry into the CNS (see the figure).

Mouse models of AD poorly represent the human condition. Could immune checkpoint blockade still hold therapeutic value for neurodegenerative diseases? Although there is limited data on how immunotherapy affects the human brain, anecdotal clinical reports yield little reason for optimism. In contrast to mouse models of $\mathrm{AD}$, cognitive deterioration is frequent among patients undergoing cancer treatments, including immunotherapy. Even in preclinical animal tumor models, blocking the immune checkpoint receptor CTLA-4 (combined with radiotherapy) reduced tumor growth but resulted in cognitive impairment (9). In principle, because immune checkpoint blockade enhances the overall ability of the adaptive immune system to target multiple disease-associated factors, one could explore its usefulness in neurodegenerative diseases other than $\mathrm{AD}$.

In prion diseases, however, targeting the immune checkpoint molecules PD1 and LAG3 did not alter prion deposition and the course of disease $(5,6)$. Moreover, loss of PD1 did not induce myeloid mobilization to prion-infected brains (6). These results again suggest that PD1 inhibition may not suffice to promote the entry of peripheral myeloid cells into the CNS. Therefore, current evidence does not encourage the direct testing of immune checkpoint blockade in patients affected by neurodegenerative diseases. However, the increasing adoption of immune checkpoint inhibition against a growing variety of common cancers will enable appropriately powered investigations of the effects of immunotherapy on human brain functions. Similarly, patients are increasingly subjected to anti-cancer immunotherapy, and some of them are likely to develop neurodegenerative diseases as they age. Analyzing the progression of disease in these patients, including neuropathological assessments of autopsied brains, may provide much-needed answers to these critical questions. 
In a parallel narrative, growing evidence suggests that targeting the innate arm of immunity inside the brain may hold more promise than targeting its adaptive arm through systemic immune checkpoint blockade. The biggest surprise delivered by genome-wide association studies of neurodegeneration is the correlation of neuroinflammatory genes in these diseases (10). Many biological processes can lead to the activation of astrocytes and microglia (non-neuronal cells in the brain), which in turn can be beneficial or deleterious. Hence, identifying specific pathways for intervention is crucial for the success of therapies targeting non-neuronal cell reactions in neurodegenerative diseases.

Perhaps the most promising progress in this aspect is the discoveries of inflammasome activation in $\mathrm{AD}, \mathrm{PD}$, and other similar diseases such as amyotrophic lateral sclerosis (11-13). Inflammasomes are large complexes that play key roles in the innate immune responses and in the maturation and secretion of proinflammatory cytokines, such as interleukin-1 $\beta$ (IL-1 $\beta$ ). An inflammasome consists of a sensor molecule, such as NOD, LRR- and pyrin domain-containing 3 (NLRP3), the adaptor protein apoptosis-associated speck-like protein containing CARD (ASC), and caspase-1, which together form a functional complex upon infection, tissue damage, or protein misfolding, such as $A \beta$ pathologic aggregation.

In the brains of patients affected by $\mathrm{AD}$ and PD, the inflammasome is strongly activated within reactive microglia $(11,12)$. The cascade of inflammasome activation in these cells leads to the generation of ASC "specks," large assemblies of ASC protein that can selfpropagate (much like prions), driving the neurotoxic inflammatory response and accelerating $\mathrm{AD}$ pathology by acting as seeds for further $A \beta$ aggregation $(11,14)$. Genetic inhibition of inflammasome activity reduces not only the $A \beta$ load, but also proinflammatory cytokine production and cognitive impairment in mouse models of AD (11). Similarly, oral treatment with a small molecule inhibitor of NLRP3 inflammasome prevented $\alpha$-synuclein pathology and dopaminergic neurodegeneration in PD mouse models, resulting in improved motor function (12).

Furthermore, several non-steroidal antiinflammatory drugs (NSAIDs) of the fenamate class are effective inhibitors of NLRP3 inflammasome activation (15). Treatment with fenamate NSAIDs restored the cognitive function in a mouse model of
AD (15). These studies have laid a solid foundation for the clinical translation of inflammasome inhibitors and repurposing fenamates to treat $\mathrm{AD}$ and potentially other forms of neurodegeneration.

Although at first they may seem contradictory, these findings point to neuroinflammation as a driver of AD and possibly of other neurodegenerative conditions. These developments herald a paradigm shift, away from the "amyloid cascade" hypothesis, which posits that in neurodegenerative diseases, pathologies stems from protein aggregation. Although nearly all therapeutic attempts are still aimed at quenching protein aggregation, it is increasingly evident that aggregation is only a part of the story. How the pathological accumulation of aggregated proteins affects innate (and perhaps adaptive) immunity, and vice versa, will certainly be addressed by future studies. Identifying the receptor, adapter, and effector molecules involved in this crosstalk may represent the best path forward to identify therapies that are more effective than those hitherto tested.

\section{REFERENCES AND NOTES}

1. M. A. Klein et al., Nature 390, 687 (1997).

2. K. Baruch et al., Nat. Med. 22, 135 (2016).

3. M. Schwartz, Science 357, 254 (2017).

4. M. Latta-Mahieu et al., Glia 66, 492 (2018).

5. Y. Liu, S. Sorce, M. Nuvolone, J. Domange, A. Aguzzi, Sci. Rep. 8, 14600 (2018).

6. J. Obst, R. Mancuso, E. Simon, D. Gomez-Nicola, Brain Behav. Immun. 73, 708 (2018).

7. X. Mao et al., Science 353, 6307 (2016).

8. L. M. Boulanger, Neuron 64, 93 (2009).

9. G. J. McGinnis et al., Oncotarget 8, 9155 (2017).

10. R. Sims et al., Nat. Genet. 49, 1373 (2017).

11. M. T. Heneka et al., Nature 493, 674 (2013).

12. R. Gordon et al., Sci. Transl. Med. 10, eaah4066 (2018).

13. S. Johann et al., Glia 63, 2260 (2015).

14. C. Venegas et al., Nature 552, 355 (2017).

15. M. J. Daniels et al., Nat. Commun. 7, 12504 (2016).

ACKNOWLEDGMENTS

A. A. is supported by the European Research Council, the Swiss National Research Foundation and the Nomis Foundation. 